\title{
Pore structure determination in bakery products by magnetic resonance imaging
} Regier, M. ${ }^{* 1}$, Schuchmann, H.P. ${ }^{1}$, Hardy, E.H. ${ }^{2}$

${ }^{1}$ Insitute of Process Engineering in Life Sciences, Department of Food Process Engineering, University of Karlsruhe, 76131 Karlsruhe, Germany

${ }^{2}$ Institute of Mechanical Process and Mechanical Engineering, University of Karlsruhe, 76131 Karlsruhe, Germany

* Corresponding author: marc.regier@LVT.uni-karlsruhe.de

\begin{abstract}
A lot of processes in food technology are mainly controlled by temperature- and waterdistributions, both determining the final product quality. In the case of fermentation and baking of doughs an additional parameter of relevance is the three-dimensional pore structure. It may be influenced by the ingredients (dough recipe) as well as by the dough preparation, fermentation and baking process.

Conventional methods for the local determination of temperatures, water concentration and binding and/or the pore size respectively the wall size distribution are mostly invasive or require elaborate sample preparation. Most of them give two-dimensional data only.

An alternative method is given by magnetic resonance imaging (MRI), which combines the advantages of being non-invasive, with sufficient time-, temperature- and local resolution.

The MRI method and results will be depicted in this presentation. Its applicability to real food systems and processes will be presented using the example of pore structure determination in bakery products.

In this work the development of the porous structure during fermentation and baking of a yeast dough is studied inline in the processes. The increase in pore volume and dough structure has been investigated. For a finished product three-dimensional pictures of the pore and wall size distribution are created. After the implementation of an algorithm analyzing the three-dimensional MRI data, pore size distributions could be determined.
\end{abstract}

\section{Materials and methods}

Dough and products investigated

The dough has been produced using $100 \mathrm{~g}$ of wheat flour (Type 405), $60 \mathrm{~g}$ of demineralised water, $5 \mathrm{~g}$ of sugar and $2 \mathrm{~g}$ of dry yeast powder as well as traces of a gadolinium-complex, reducing the relaxation time and thus the NMR measurement time. The dry yeast powder has been distributed within the wheat flour, adding the water $\left(30^{\circ} \mathrm{C}\right)$, and kneaded by hand for approximately $5 \mathrm{~min}$.

A small sample of the dough of approximately $1 \mathrm{~g}$ was spherically formed, filled into a glass tube of $1.5 \mathrm{~cm}$ outer diameter and introduced into the MRI tomograph.

As an example of a finished product, a piece of commercial bread called 'Hüttenbrot' from a local bakery was investigated.

\section{Magnetic Resonance Imaging (MRI)}

For all experiments a Bruker Super Wide Bore Kryo-magnet (Bruker Biospin, Rheinstetten) with a magnetic field of $4.7 \mathrm{~T}$ (proton resonance frequency $200 \mathrm{MHz}$ ) and a bore of $150 \mathrm{~mm}$ has been used. It is equipped with a Bruker Avance 200-console and imaging unit. Different 
gradient units with inner diameters of up to $64 \mathrm{~mm}$ may be used allowing magnetic field gradients for imaging of up to $1 \mathrm{~T} / \mathrm{m}$.

For the investigations on the dynamical changes of pore structure during fermentation and baking, the tomograph was equipped by a probe, which is temperature controlled by gas convection between $-100{ }^{\circ} \mathrm{C}$ and $180{ }^{\circ} \mathrm{C}$ (Micro2.5EVT, Bruker Biospin, Rheinstetten). A Multi Slice Multi Echoe (MSME)-sequence was chosen with $128 * 128 * 5$ volume elements (voxels), where one slice had the dimensions of $2 \mathrm{~cm} * 2 \mathrm{~cm}$ and a thickness of $1 \mathrm{~mm}$. Two to four averages have been used, the echo time of approximately $3 \mathrm{~ms}$ and repetition time of 230 ms resulted in measurement times of approximately 1 and 2 min, respectively.

The 3D MRI data of the finished bread was acquired by a 3 dimensional stimulated echo sequence. As antenna, an open-ended Bruker bird-cage probe head with $15 \mathrm{~mm}$ inner diameter was used. That is why the bread has been cut into pieces of a corresponding size with a sharp knife, taking care for not changing pore volumes. NMR pulse sequences have been used as provided by Bruker. For measuring the 3D proton density distributions which correspond to the dough walls, a stimulated echo pulse sequence has been used. A volume of $15 \mathrm{~mm} * 15 \mathrm{~mm} * 15 \mathrm{~mm}$ has been divided into $256^{3}$ voxels, corresponding to an isotropic resolution of $58.5 \mu \mathrm{m}$. The MRI-data obtained (3D-matrices) has been exported for further analysis.

\section{Algorithm for determining pore size distributions}

The obtained 3D data was converted into Matlab ${ }^{\mathrm{TM}} 6.5$ (The MathWorks, Inc.) matrices for analysis of the pore-space structure. After choosing the region to be analysed (a cuboid) the automated meaningful decomposition of the pore space into individual pores is crucial.

The first step is to build a binary matrix from the input intensity data by setting a suitable threshold value; intensities below this threshold are set to 0 , intensities above are set to meaning a voxel filled by wall material. This threshold has to be chosen carefully by finding the minimum in the intensity distribution diagram, so that the macroscopic porosity agrees with an independently obtained value.

The next step is to find the pore voxels, which have at least one contact plane to a wall voxel. These are marked to have the height 1 and are defined to newly be filled by material. In this new matrix, again the pore voxels, which have a contact plane with the new walls are marked to have height 2 and are now filled by material. The procedure is carried out, until all pixels are filled by materials and are assigned to a defined height. This algorithm is known as total thinning [Baldwin et al., 1996].

The third and critical step is the decomposition of the matrix obtained in the previous step into individual pores. At the beginning centres for the pores are identified. The centres are local maxima in the matrix with the heights corresponding to the thinning steps. The range for the definition of local maxima has to be defined carefully so that even for small pores centres are found. For larger pores with several maxima, the most appropriate has to be determined. In the following, the remaining pore-space voxels have to be assigned to the corresponding centres. If this is done suitably, clean separations between connected pores are obtained. Details will be published in a forthcoming paper.

In order to determine the volume of each pore, the number of voxels belonging to each pore centre are counted, respectively and are multiplied by the volume of one voxel.

The pore size density distribution as a volume density distribution is then calculated by assigning the pores to classes of different size, counting the numbers within the classes and normalising to the total number of pores and the class width. Whereas the number of size classes $\mathrm{k}$ is defined by equation 1 [Richter, 2002], the class widths can be chosen as a constant value or as a term of a geometric progression, what gives more detailed information for smaller pore sizes. 
$k=\operatorname{integer}\left\{\begin{array}{cc}\sqrt{n} & , n \leq 100 \\ 5 \cdot \log n & , n>100\end{array}\right.$

By integration of the density distributions over the pore volume the cumulative size distributions are obtained.

As additional information, for 3D data the surface to volume ratio of the pores can be calculated. This is done by dividing the number of voxels with height 1 (weigthed by the number of contact planes to the surrounding wall voxels) to the number of all pore voxels and multiplying with the ratio of one voxel plane to the voxel volume.

\section{Results and discussion}

Dynamic inline observation of yeast dough fermentation and baking

After the production of the dough and introduction into the tomograph, a first magnetic resonance image (central vertical slice) of the dough has been acquired. During the first 15 minutes the convective airflow temperature was controlled to stay at $17^{\circ} \mathrm{C}$. As can be seen in figure 1 the dough is nearly unstructured, showing only a few pores to be resolved. After heating up to $30^{\circ} \mathrm{C}$ (after $15 \mathrm{~min}$ ), the fermentation controlled generation of $\mathrm{CO}_{2}$ and thus growth of bubbles in the dough could be observed (Fig.1) in two-dimensional pore density image (after $25 \mathrm{~min}$ ). The volume expanses further as can be observed in the images for 43 min and $45 \mathrm{~min}$, showing two rather large pores at the right side. These pores strongly expand, coalesce and disrupt the outer dough membrane before collapsing. By heating the airflow up to $150^{\circ} \mathrm{C}$ after $60 \mathrm{~min}$, the also baking process could be followed inline showing a further pore structure forming and volume expansion. After approximately $64 \mathrm{~min}$ the maximum dough volume was reached, the further baking process yields an intensified drying, what is the reason for a total volume reduction or shrinkage.

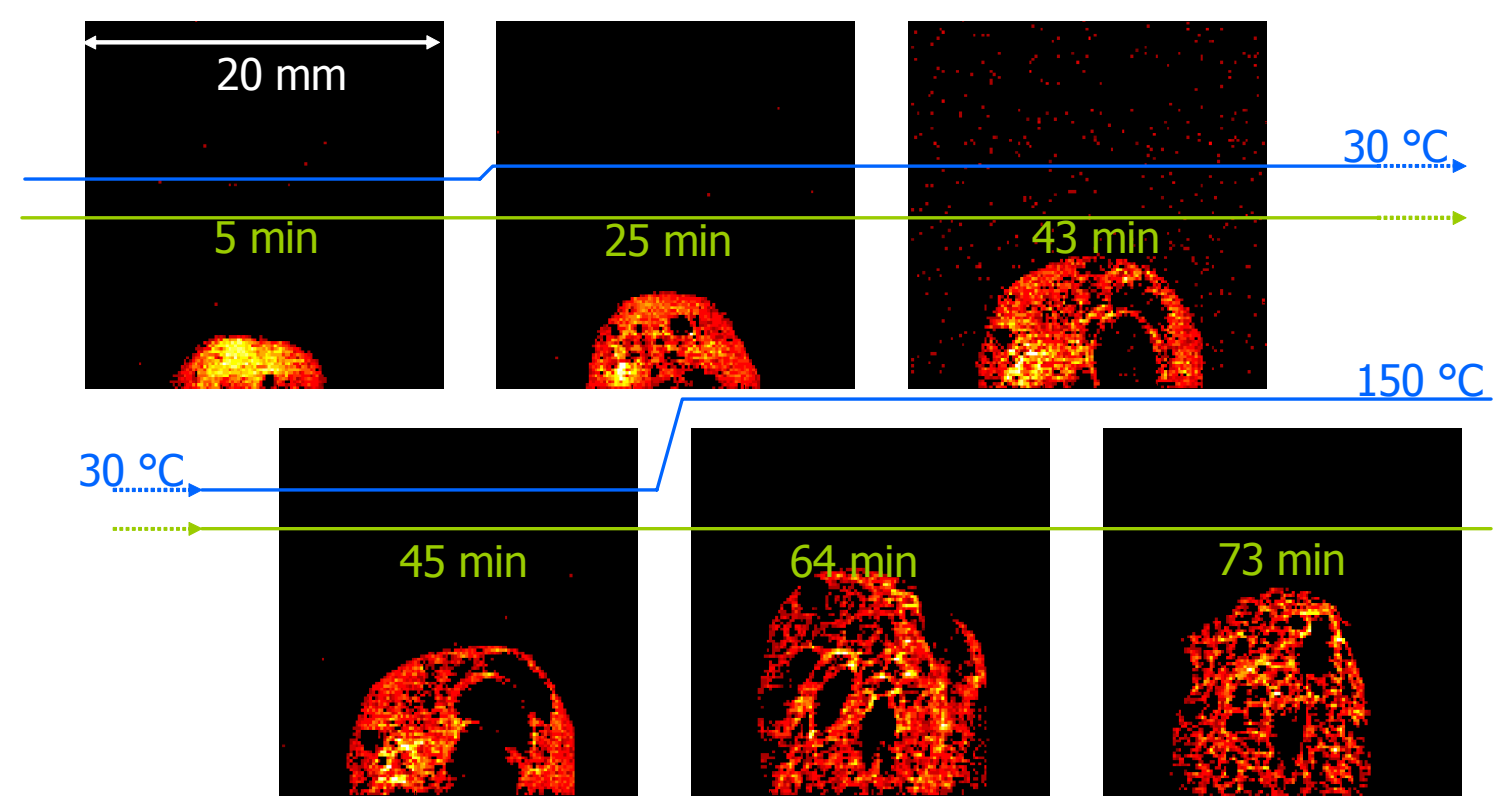

Fig. 1: Sequential MRI-images of the fermentation and baking of a yeast dough. 


\section{Pore size distribution determination of finished products}

Figure 2 shows the 3D MRI data of the bread as a surface reconstruction as well as a scanner image of one slice of the same product. The 3D data has been analysed by the pore size distribution determination algorithm as mentioned above.

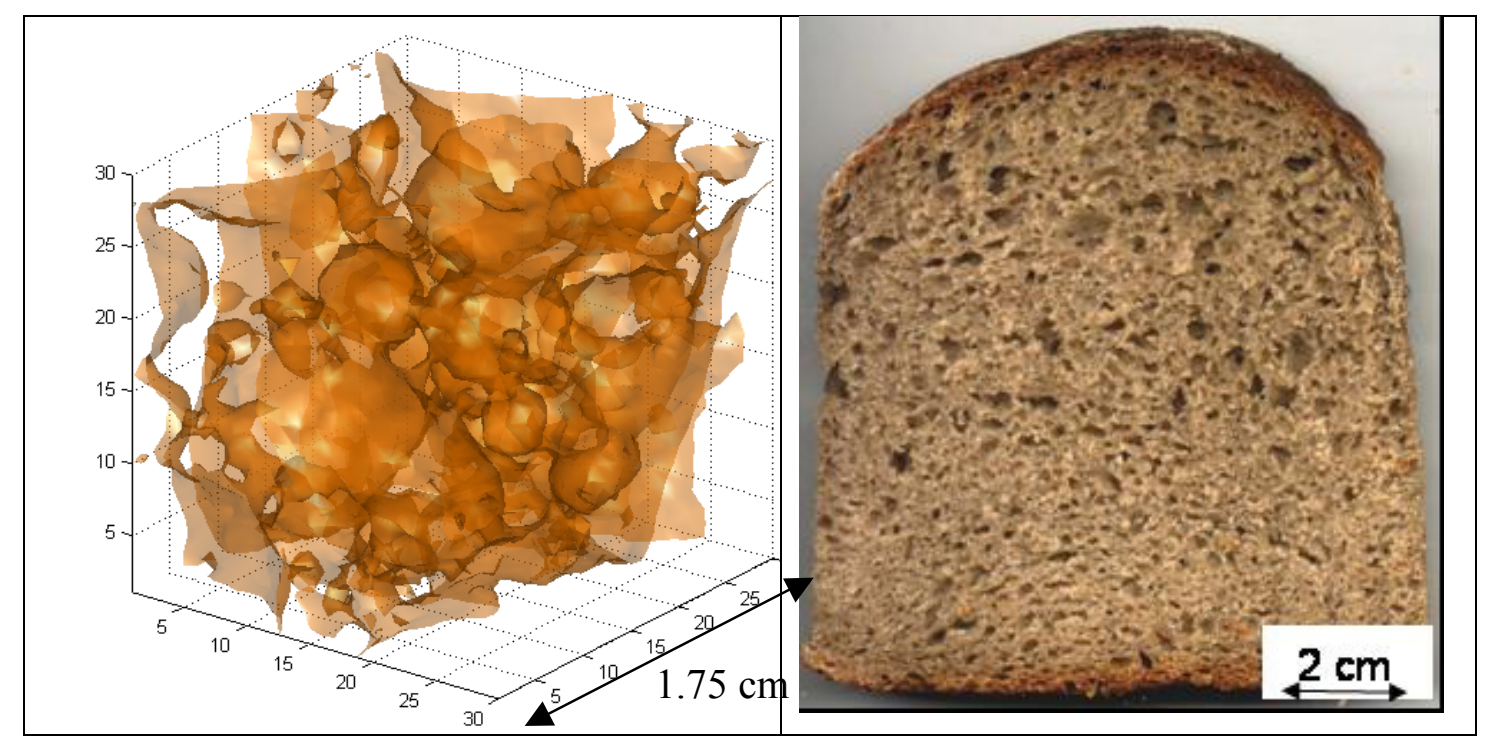

Figure 2: 3D surface reconstruction of the pore space of "Hüttenbrot" and a scanner image of a slice of the same product.

The results are presented in figure 3 in comparison to the estimated pore volume distribution from image analysis of 2D scanner images. Both the volume density as well as the cumulative volume distribution are given.

Obviously the results agree rather well with each other above pore volumes of approximately $0.3 \mathrm{~mm}^{3}$. Below this value, the local maximum frequency has shifted by one dimension from $3 \cdot 10^{-3} \mathrm{~mm}^{3}$ (from MRI-data) to $3 \cdot 10^{-4} \mathrm{~mm}^{3}$ (from scanner data). This points to the problems of the evaluation of 2D-images: The slice of bread and thus also the pores have to be cut, so that the $2 \mathrm{D}$ projection is much smaller than the mean pore cutting area. On the other hand, the smallest detected pore volumes are also smaller for scanner data than for MRI data, due to the fact that the local resolution of our MRI data was limited (by our experiment) to approximately $60 \mu \mathrm{m}$ compared to a maximum resolution of the scanner of ca. $3 \mu \mathrm{m}$.

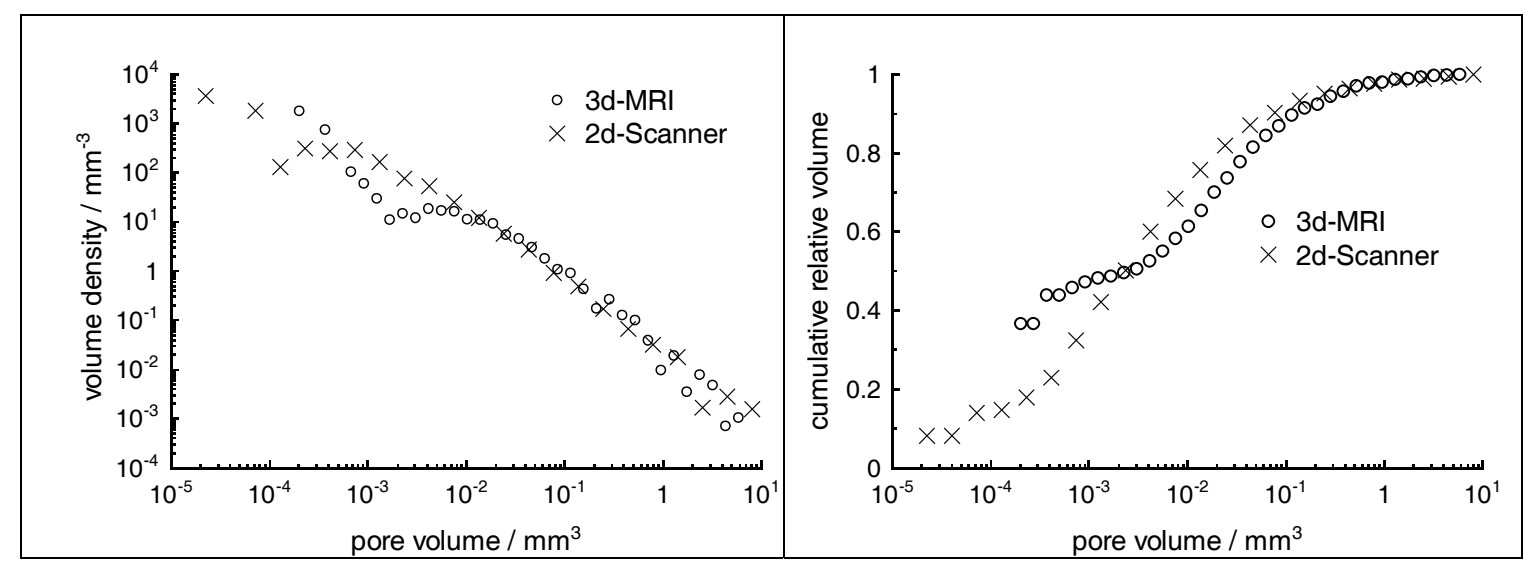

Figure 3: Comparison of pore volume distributions of bread as analysed from real 3D-MRIdata and estimated by $2 \mathrm{D}$-scanner-image analysis. 
As additional result of the analysis of MRI data, the surface to volume ratio $\mathrm{S} / \mathrm{V}$ of the pore space can be calculated as explained in the materials and methods section. For the investigated bread this value has been determined to be: $\mathrm{S} / \mathrm{V}_{\text {pore }} \approx 23422 \frac{1}{\mathrm{~m}}$. When relating the pore surface not only to the pore volume but to the whole volume investigated, the surface to volume ratio calculates to:

$$
\mathrm{S} / \mathrm{V}_{\text {total }} \approx 16372 \frac{1}{\mathrm{~m}}
$$

These data may serve in future investigations for the determination of process functions determining the correlations between recipes and/or process parameters and pore size distributions as well as property functions describing the correlations between pore size distributions and product properties (sensorial, aging,...). Product and process functions will then be the basis for a model based optimisation of product recipes and process parameters

\section{Acknowledgements}

The authors thank the DFG for making the tomography available, Kai Knoerzer for helpful discussions as well as Emilio Oliver-Gonzalez for technical support.

\section{References}

Baldwin, C.A., Sederman, A..J., Mantle, M.D., Alexander, P., Gladden, L.F. Determination and Characterization of the Structure of a Pore Space from 3D Volume Images, Journal of Colloid and Interface Science, 181,1, 1996, 79-92

Richter, C.: Einführung in die Biometrie - 1 Grundbegriffe und Datenanalyse, ISBN 3930037-03-3, Saphir-Verlag, 2002. 